\title{
Transition from Nondestructive Testing (NDT) to Structural Health Monitoring (SHM): potential and challenges (presentation video)
}

\section{Peter Cawley}

Peter Cawley, "Transition from Nondestructive Testing (NDT) to Structural Health Monitoring (SHM): potential and challenges (presentation video)," Proc. SPIE 9055, Bioinspiration, Biomimetics, and Bioreplication 2014, $90550 Z$ (8 May 2014); doi: 10.1117/12.2049219 Health Monitoring, 2014, San Diego, California, United States 


\title{
Transition from Nondestructive Testing (NDT) to Structural Health Monitoring (SHM): potential and challenges (Presentation Video)
}

\author{
Peter Cawley, Imperial College London (United Kingdom)
}

\begin{abstract}
There is a gradual shift in emphasis from periodic inspection with detachable transducers (NDT) to permanently installed monitoring systems giving information about the structural integrity at pre-programmed intervals or on demand (SHM). The drivers of this change are discussed, together with the requirements of successful SHM systems. Particular issues are that NDT often involves scanning and this is not possible with typical SHM configurations; it therefore becomes important to cover a significant area of structure from each transducer position. Guided waves provide a possible solution to this problem and permanently installed guided wave pipe inspection systems are now available. The sensitivity obtained with a permanently installed system is significantly better than that in a one-off test as baseline subtraction can be employed. However, this is far from trivial as it is necessary to compensate for benign changes such as temperature. The guided wave technique does not provide accurate remaining thickness information and is best complemented by point measurements at selected locations. Another issue is that the SHM transducers must survive in operational conditions, which is particularly difficult at high temperatures. Recent work at Imperial College and associated spin-out companies on solutions to these problems is discussed.
\end{abstract}

View presentation video on SPIE's Digital Library: http://dx.doi.org/10.1117/12.2049219.3385681145001 